\title{
The psychophysics of the kinesthetic aftereffect in the Petrie block experiment
}

\author{
ERNEST R. HILGARD, ${ }^{2}$ ARLENE H. MORGAN, AND SUSAN PRYTULAK \\ STANFORD UNIVERSITY
}

\begin{abstract}
Kinesthetic size is reported in the Petrie experiment by a simultaneous size comparison in which a standard block held between the thumb and forefinger of one hand is matched in size by moving the thumb and forefinger of the opposite hand along a wedge until the proper width is reached. After a baseline is obtained, a kinesthetic aftereffect is produced by rubbing a block of contrasting size followed by a return to the standard block. Experiments with 22 male Ss and 20 female Ss, tested in separate cycles, show that augmentation following stimulation with a block smaller than standard and reduction following stimulation with a block larger than standard are both statistically significant, and, at least for augmentation, the aftereffect persists for at least $48 \mathrm{~h}$. When the data are corrected for regression there is no significant relationship between initial baseline and the amount of augmentation or reduction; reasons why such a relationship has been reported are indicated. The results suggest that when this kind of experiment is used for the study of individual differences $(a)$ it is satisfactory to derive augmentation and reduction scores by subtracting the baseline from the absolute scores, provided the scores are corrected for regression, and $(b)$ a counterbalanced order of presentation is not advisable in view of the carryover of augmentation from one day to the next, which is likely to make the two orders incommensurate.
\end{abstract}

Figural aftereffects, much studied in vision, have been known to apply also to kinesthetic size judgments ever since the early observations of Gibson (1933). They were more thoroughly explored by Köhler and Dinnerstein (1947), and have been studied in various contexts by others, e.g., Bakan and Weiler (1963), Bakan and Thompson (1967), Dinnerstein and others (1962), and Blitz and others (1966). Many experiments, using procedures modified from those of Köhler and Dinnerstein have been conducted by Petrie and her associates and reported in a recent book (Petrie, 1967). Her concerns have been with individual differences in the kinesthetic aftereffect as these are related to other phenomena, chiefly sensitivity to pain. In the effort to repeat and extend some of her observations, it seemed important to us to report more fully on the psychophysical aspects of her procedures to the extent that these may influence the use of the data for the study of individual differences.

Petrie's findings are essentially that there are those who magnify sensory inputs, the augmenters, and that their kinesthetic responses are predictive of their over-reaction to pain. Contrariwise, there are those who minimize sensory inputs, the reducers, and their underestimation of kinesthetic size (under appropriate conditions of contrast) predicts their high tolerance for pain. The logic of the test is, of course, that the augmenters depart from the norm in one direction and reducers in the opposite direction, thus constituting the two extremes (approximately the upper and lower thirds) of a distribution. In this paper we shall not be testing these conjectures of the relationship between kinesthetic effects and pain, but shall be reporting only upon certain internal aspects of the kinesthetic experiment.

\section{Procedure}

The procedure we used followed as faithfully as possible that outlined by Petrie (1967, pp. 107-127). The details will not be repeated, but the essential features are that two days of testing are separated by not less than $48 \mathrm{~h}$. We shall first indicate what the two sessions have in common, and then note their differences.

On both days, after an initial $45 \mathrm{~min}$ rest in which the fingers are not used, the $S$ is blindfolded and a baseline measure is obtained. During the rest interval the $S$ is occupied with personality tests. The experimenter recorded the verbal replies so that the $S$ did not have to use his hands. The results of these tests are irrelevant to this report. During the time that a standard block is rubbed between the thumb and forefinger of the right hand (for a right-handed person), the left hand is simultaneously moved along a wedge from the small end until a width is reached that is judged equal to the width of the block held by the right hand. The equipment includes a sliding hand and finger rest, which moves smoothly along the blocks and wedge as the thumb and forefinger rub the block or move along the wedge. Thus there is no ambiguity in reading the exact position of the fingers on the wedge, according to the location of the slide on a scale attached to the wedge. This may be considered a variant of the method of adjustment, in which the adjustment is always made in ascending order. This procedure is repeated six times, the first two measures being considered practice, while the next four are recorded. Note that the judgments are simultaneous ones, the right hand on the standard block, the left hand on the wedge. There are a number of special precautions in handling the Ss which were recommended by Petrie, and followed by us, which can be found in her book.

Measurements beyond the baseline are taken after intervening experiences which provide contrasts. The intervening experience consists of rubbing another block with the right hand, a block either larger or smaller than the standard one. The block is rubbed for $90 \mathrm{sec}$ and then another measurement is taken precisely as in the baseline period. That is, the rubbed block is replaced by the original standard one, and the left hand again tries to match the width that the right hand feels. Four such matchings are again recorded, always moving from the small end of the wedge until the $S$ is satisfied with his match. There follows another $90 \mathrm{sec}$ of rubbing the block of contrasting size, after which four more matches are obtained between the wedge and the original (standard) block. A final rubbing, this time of $120 \mathrm{sec}$ duration, is again followed by the usual four matchings of wedge and standard block. A 15-min rest follows these matchings-after-contrast; then the baseline matching is repeated four times as a test of recovery from the earlier contrasts.

Our measurements were made in two cycles, the first with a sample of male $S s(N=22)$ from an introductory psychology class, the second by a sample of female $S s(N=20)$ from the same course given in the following quarter. Because we are here primarily interested in the central tendencies, the two samples are combined for most measurements, but separate treatment will be made when desirable. The same samples were used in a study involving other measurements of pain and hypnosis, but these other comparisons, which depend upon the individual difference features, will not be treated here.

The experimental design, as it finally turned out, is presented in Table 1, which also gives the actual sizes of the standard blocks and the contrasting blocks. In the subsequent tables and figures, differences are presented in per cent, to correct for the differences in the standards. Unfortunately, there is no clear way to correct for the differences in the ratios of contrasting and standard blocks in the augmenting and reducing conditions.

\section{Results}

Having followed a procedure dictated by the requirements of an experiment designed to assess individual differences in a standardized manner, we were not free to make modifications in design that might have answered some of our questions more precisely. Therefore this analysis is based on the asking of questions of the data after they have been gathered in the standard manner. The results will be presented in the form of questions asked of the data, with answers given by such relevant data as can be found. 
Table 1

Experimental Design

\begin{tabular}{lccc} 
& Order $\mathbf{A}_{1} \mathbf{R}_{2}{ }^{*}$ & Order $\mathbf{R}_{1} \mathbf{A}_{2}$ & Total \\
\hline Male Subjects & $\mathrm{N}=10$ & $\mathrm{~N}=12$ & $\mathrm{~N}=22$ \\
Female Subjects & $\mathrm{N}=9$ & $\mathrm{~N}=11$ & $\mathrm{~N}=20$ \\
\hline Total & $\mathrm{N}=19$ & $\mathrm{~N}=23$ & $\mathrm{~N}=42$ \\
\hline
\end{tabular}

* $A$ and $R$ refer to Augmentation and Reduction, and the subscripts to the days of experimentation.

Augmentation (A): Contrast by rubbing smaller block $(25.4 \mathrm{~mm}$ ) following standard block of $50.8 \mathrm{~mm}$.

Reduction (R): Contrast by rubbing larger block $(63.5 \mathrm{~mm})$ following standard block of $38.1 \mathrm{~mm}$.

1. Are the kinesthetic contrast effects in the predicted direction for the sample as a whole? The effects in which Petrie is most interested are the departures from the average effect by those individuals who are classified as augmenters or reducers, but we are asking a different question, namely, what is the size of the mean effect apart from individual differences? When a smaller block is used as a contrast stimulus, we expect the size estimation of the standard block to be too large (Condition A, for augmentation), and when a larger block is used as a contrast stimulus, we expect the size estimation of the standard block to be too small (Condition R, for reduction). Because augmentation and reduction are deviations from standard blocks differing in size (although always uniform within conditions), we have expressed the departures as per cents of standard in order to make the changes more nearly comparable. The mean results for the two sessions are shown in Figs. 1 and 2.

Let us consider Fig. 1 first, with the results for the first session. The baseline shows a negative constant error, a result of always measuring in the ascending order on the wedge. The estimated baseline falls a mean of $7.5 \%$ below the true baseline, a difference that is statistically significant $(t=3.14$, $\mathrm{df}=41, \mathrm{p}=.01)$. This underestimation in this order has been reported by Blitz and others (1966). After the rubbing of the contrasting blocks the effects are indeed in the expected direction. The mean of the three estimates after contrast stimulation for Condition $A_{1}$ (augmentation) is $18.6 \%$ above the estimated baseline, a result that is significant at the .001 level (see Table 2). Correspondingly, the contrast stimulation for Condition $R_{1}$ (reduction) leads to a reduction of $9.0 \%$ below the baseline estimate, also significant at the .001 level.

Turning now to Fig. 2, which gives the corresponding data when the groups have had their conditions reversed on the second session (after a minimum of $48 \mathrm{~h}$ ), we note a number of differences. First, the baselines reflect the persistence of contrast effects from the first day. That is, those who experienced Condition $A_{1}$ on the first day begin with a high baseline on the second session, when they are to be in Condition $R_{2}$, while those who experienced Condition $R_{1}$ do not have elevated baselines. It is puzzling that the initial baseline for Condition $R_{2}$ (prior to any experience of contrast on the second day) is actually higher than it was at the end of Condition $A_{1}(t=2.63$, $\mathrm{df}=18, p=.02)$. Unfortunately, the new baseline is obtained with a standard block that is smaller than the standard of the first day $(38.1 \mathrm{~mm}$ vs 50.8 $\mathrm{mm}$ ) so that an equal absolute over-estimation would correspond to an even greater per cent over-estimation. It turns out, in fact, that in absolute terms the over-estimation on the second session is not greater than it was at the end of the first session $(t=1.61$, $\mathrm{df}=18$, n.s.). The contrast conditions are too complex to interpret these findings with confidence, except to point out that there is indeed some residue from the augmentation of the first session. This persistence over time is to be expected from the earlier work of Köhler and Dinnerstein (1947). It is clear, therefore, that the two sessions are not independent replications.

The mean changes after the three periods of contrast stimulation in the second session are given in Table 3. Again there is significant augmentation over the estimated baseline in Condition $A_{2}$, and significant reduction in Condition $R_{2}$. It will be noticed that on Day 1 there was a tendency toward more augmentation than reduction (Table 2) while on Day 2 there was a tendency for more reduction than augmentation (Table 3). It is not possible to say with assurance whether or not this is a consequence of the order or a consequence of differences in the $A_{1} R_{2}$ and $R_{1} A_{2}$ samples; only further experimentation with a more adequate design can find this out.

A point to be noted also with respect to Fig. 2 is that by the end of the second session, having experienced contrasts in both directions, the mean result is that the average size estimate approaches very close to the actual size; that is, the size judgments are becoming more accurate, or at least, the average constant error is becoming very small. ${ }^{3}$

We have thus demonstrated both that the contrast effects are in the expected direction, and that the contrast effect, particularly for augmentation, persists for at least $48 \mathrm{~h}$.

2. Is the baseline on the first day predictive of later augmentation or reduction? Petrie (1967, pp. 135-140), noting that augmenters generally have lower baselines than reducers, gives an explanation in terms of the stimulation provided by moving the hand along the wedge in making the measurements, reducers therefore moving too far along in establishing their baselines, and augmenters stopping short because they have already magnified the stimulus. Thus she says, "Indeed, with certain important exceptions one can start identifying the reducer from the larger

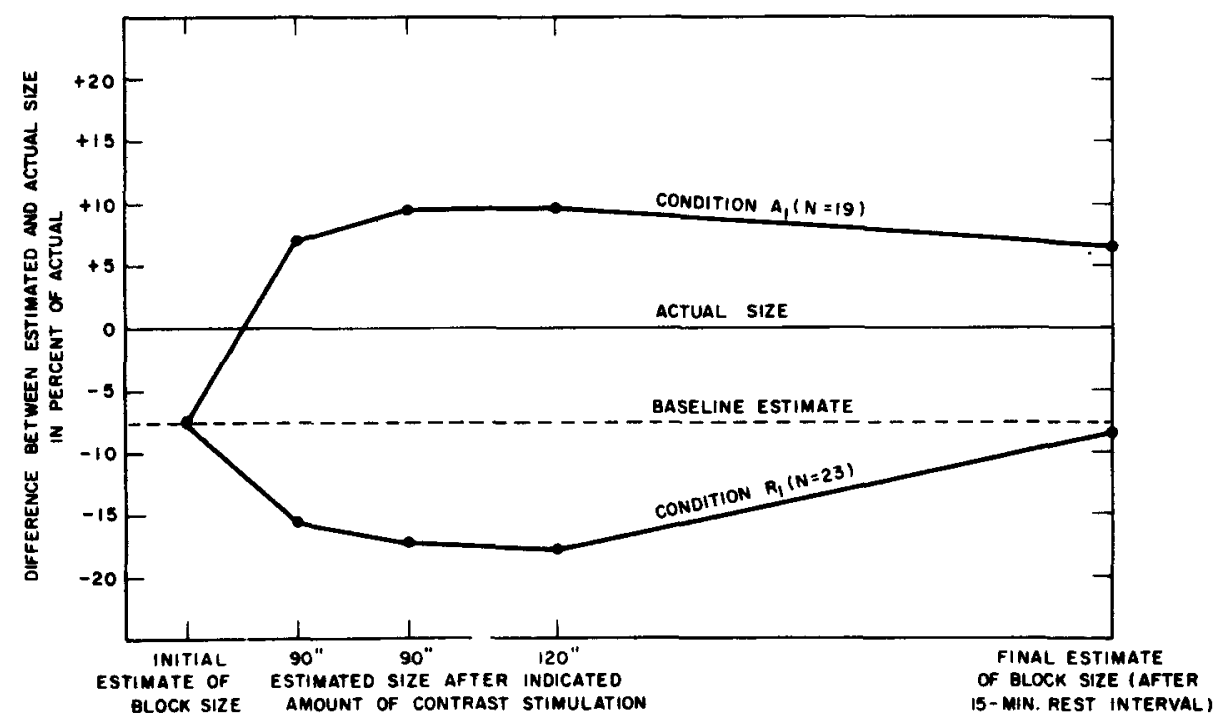

Fi. 1. The course of augmentation (Condition $A_{1}$ ) and of reduction (Condition $R_{1}$ ) on the initiel day of experimentation. 


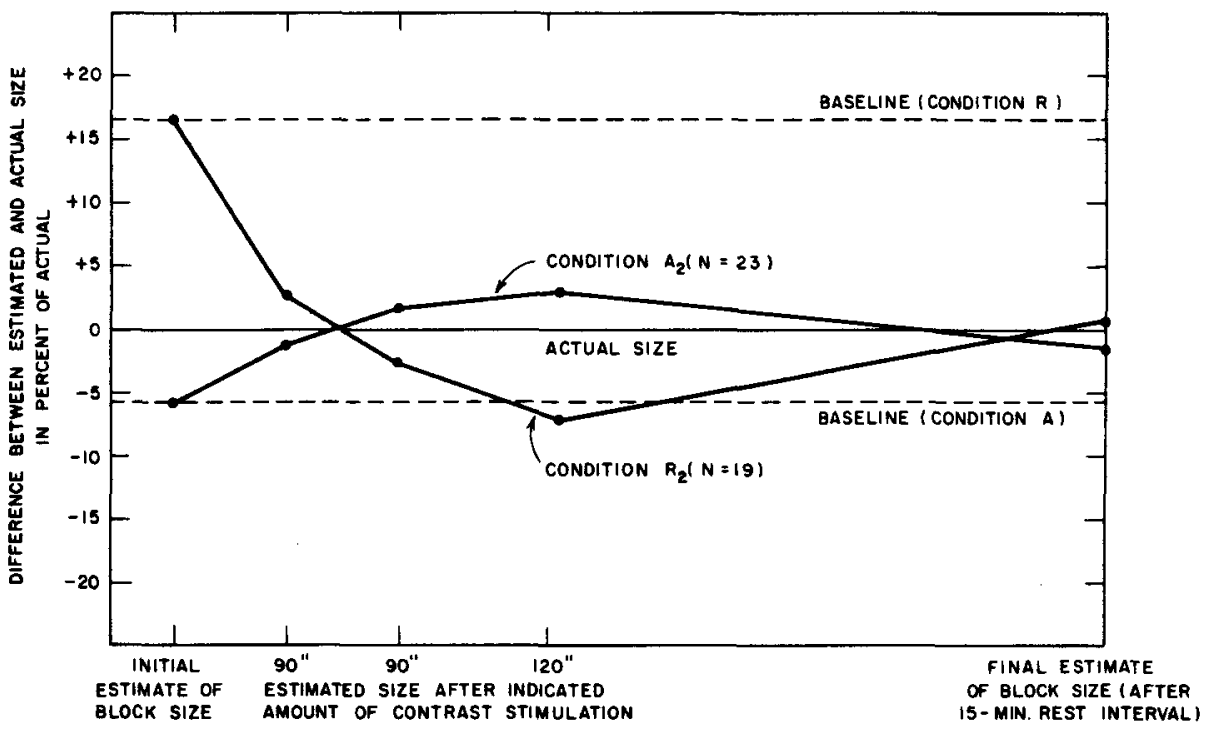

Fig. 2. The course of augmentation (Condition $\mathrm{A}_{2}$ ) and of reduction (Condition $R_{2}$ ) on the second day of experimentation, indicating aftereffects carrying over from other conditions on first day.

baseline measurements that he provides" (p. 136). Unfortunately, augmentation and reduction are measured as difference scores from the baseline, so that, with fallible measures, any $S$ who happens to make a high baseline score is more likely to end up with a reduced score, and any $S$ who happens to make a low baseline score is more apt to end as an augmenter. This is the familiar fact of regression, sometimes stated as the "law of initial value," producing a negative correlation between initial score and gains.

A second circumstance which produces a tendency for augmenters to have low baselines and for reducers to have high baselines is that half of them begin their augmentation on Day 2 and half begin their reduction on Day 2 , a session in which, as a result of the prior experience, the reducers start from a low baseline and the augmenters from a high baseline, as shown in Fig. 2. How this affects the averages (half having had the order $A_{1} R_{2}$ and half having had the order $R_{1} A_{2}$ ) is shown in Fig. 3. The sexes are treated separately, in order to indicate the degree to which the effects are replicated. It is evident that augmentation tends to start from a low baseline and reduction from a high one, but these average effects have nothing to do with individual tendencies to augment or to reduce beyond the demands of the stimulus constrasts.

It may be conjectured that those who are prominently augmenters will tend to reflect what is found in general under augmentation, and those who are reducers will reflect what is found in general under reducing conditions. Hence, it will take some careful analysis to see what the psychological conditions are that determine the relationship between baseline and reduction or augmentation, as distinct, first, from statistical regression, and second, from the artificial effect on baselines of the carry-over contrast effects between days.

The appropriate method for starting this analysis is to confine ourselves to the first session only, thus eliminating the artificial

Table 2

Augmentation and Reduction within Day 1

Differences from Estimated Baseline as Per Cent of Standard

\begin{tabular}{llrr}
\cline { 3 - 4 } \multicolumn{1}{c}{ Contrast Conditions } & $\mathrm{n}$ & mean & S.D. \\
\hline Condition $\mathrm{A}_{1}$ (augmentation) & 19 & +18.6 & 11.8 \\
Condition $\mathrm{R}_{1}$ (reduction) & 23 & -9.2 & 9.5 \\
\hline
\end{tabular}

Significance tests:

Condition $A_{1}$, augmentation from baseline, df $18, t=6.86, p=.001$.

Condition $R_{1}$, reduction from baseline, df $22, t=4.65, p=.001$. influence of the kinesthetic after-effects of the first session upon the baselines of the second session. Then we seek to correct, if possible, the regression effects, so that those with lower baselines do not necessarily "augment" more, and those with higher baselines do not automatically "augment" less.

It turns out that for Condition $A_{1}(N=19)$ the correlation between the baseline on Day 1 and the third measurement (after a total of $300 \mathrm{sec}$ of contrast stimulation) is $r=.83$, and the correlation between the baseline and the amount of regression is $r=-.02$. Thus there is very little regression involved, in the sense that the gains are shown not to be functions of the initial scores. If a correction is made for the expected regression based on the correlation between initial and final scores (see McNemar, 1962, pp. 158-161), the corrected correlation between baseline and gain ("augmentation") becomes a nonsignificant $r=.23$. This means, if this positive correlation were to be significant, there would be a higher augmentation for those with initial high baselines, a conclusion opposite to that proposed by Petrie, but as things stand we may simply say that there is no established relationship at all.

For Condition $R_{1}(N=22)$ the correlation between baseline and the third reduction measure (after a total of $300 \mathrm{sec}$ of contrast stimulation) is $r=.65$ and the correlation between baseline and score changes (reduction) is $r=-.45$, indicating that the higher the baseline the greater the reduction, as implied by Petrie. However, this conclusion is subject to correction for regression based on the correlation between baseline and final score $(r=.65)$, by the method referred to. When thus corrected the correlation between the baseline and the amount of reduction becomes $r=-.02$, a nonsignificant value, agreeing with the finding for augmentation.

It may be asserted with some confidence, therefore, that there is no relationship between the original baseline and the tendency to augment or to.reduce, and that the results which are found to show a relationship are artifacts in the treatment of the data.

Table 3

Augmentation and Reduction within Day 2

Differences from Estimated Baseline as Per Cent of Standard

\begin{tabular}{lccr}
\multicolumn{1}{c}{ Contrast Conditions } & $\mathrm{n}$ & mean & S.D. \\
\hline Condition $\mathrm{A}_{2}$ (augmentation) & 23 & +8.6 & 10.9 \\
Condition $\mathrm{R}_{2}$ (reduction) & 19 & -15.0 & 8.3 \\
\hline
\end{tabular}

Significance tests:

Condition $A_{2}$, augmentation from baseline, df $22, t=3.79, p=.001$.

Condition $R_{2}$, reduction from baseline, df $18, t=7.88, p=.001$. 


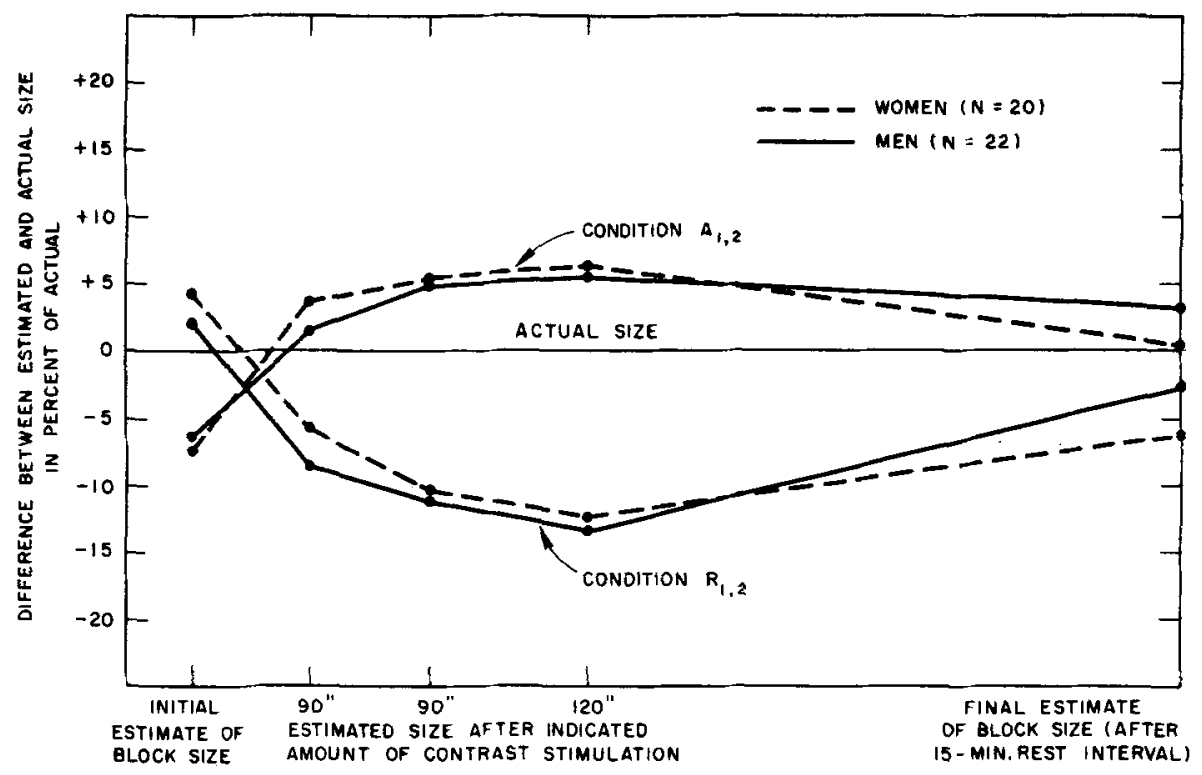

Fig. 3. Mean results for augmentation (Condition $A_{1,2}$ ) and reduction (Condition $R_{1,2}$ ) by sex, indicating artificiality of baselines (initial estjmate) as a consequence of change in conditions between days.

\section{Conclusions}

1. Kinesthetic contrast effects are demonstrated significantly in the standard artangements of the Petrie experiment. That is, the standard block is judged larger after stimulation with a smaller one, and smaller after stimulation with a larger one.

2. The contrast effect, particularly for augmentation after stimulation with a block smaller than the standard, persists for at least $48 \mathrm{~h}$, and results in a modified baseline on the second day for those who experienced augmentation on the first.

3. When scores are corrected for the effects of regression, there is no evidence that later augmentation or reduction is a consequence of initial baseline levels. When thus corrected, therefore, difference scores from the baseline can be used satisfactorily as a measure of the contrast effects.

The analyses here reported are not concerned with the persistence of individual differences in departures from the norm, but solely with the average trends within the data. In view of the persistence of the aftereffects of augmentation, it would appear unwise, in later replications of the Petrie experiment, to use a counterbalanced order when individual differences are being assessed, because the second day measurements are then initiated from baselines which differ markedly for those whose experiences differed on the first day.

\section{REFERENCES}

BAKAN, P., \& WEILER, E. Kinesthetic aftereffect and mode of exposure to the inspection stimulus. J. exp. Psychol., 1963, 65, 319.320.

BAKAN, P., \& THOMPSON, R. W. Induction and retention of kinesthetic aftereffects as a function of number and distribution of inspection trials.
Percept. \& Psychophys., 1967, 2, 304-306.

BLITZ, B., DINNERSTEIN, A. J., \& LOWENTHAL, M. Relationship between pain tolerance and kinesthetic size judgment. Percept. mot. Skills, $1966,22,463-469$.

DINNERSTEIN, A. J., LOWENTHAL, M., MARJON, R. B., \& OLIVO, J. Pain tolerance and kinesthetic after-effects. Percept. mot. Skills, 1962, 15, 247-250.

GIBSON, J. J. Adaptation, after-effect and contrast in the perception of curved lines. J. exp. Psychol, 1933, 16, 1-31.

KÖHLER, W., \& DINNERSTEIN, D. Figural after-effects in kinesthesis. In A. Michotte (Ed.), Miscellaned psychologica. Paris: Joseph Vrin, 1947. Pp. 196-220.

MCNEMAR, Q. Psychological statistics. (3rd ed.). New York: Wiley, 1962.

PETRIE, A. Individuality in pain and suffering. Chicago: University of Chicago Press, 1967.

\section{NOTES}

1. We wish to express our appreciation to Dr. Aserath Petrie who spent several months in our laboratory supervising the training of those who did the actual testing with the block apparatus which she kindly contributed. Support for the research was provided by the National Institutes of Health, Grant MH-3859, and the U.S. Air Force Office of Research, Contract AF 49 (638)-1436.

2. Address: Department of Psychology, Stanford University, Stanford, Catif. 94305.

3. The variable errors, however, have not been very much reduced.

(Accepted for publication April 25, 1968.) 